\title{
Working memory load can both improve and impair selective attention: Evidence from the Navon paradigm
}

\author{
Lubna Ahmed • Jan W. de Fockert
}

Published online: 8 August 2012

(C) Psychonomic Society, Inc. 2012

\begin{abstract}
Selective attention to relevant targets has been shown to depend on the availability of working memory (WM). Under conditions of high WM load, processing of irrelevant distractors is enhanced. Here we showed that this detrimental effect of WM load on selective attention efficiency is reversed when the task requires global- rather than locallevel processing. Participants were asked to attend to either the local or the global level of a hierarchical Navon stimulus while keeping either a low or a high load in WM. In line with previous findings, during attention to the local level, distractors at the global level produced more interference under high than under low WM load. By contrast, loading WM had the opposite effect of improving selective attention during attention to the global level. The findings demonstrate that the impact of WM load on selective attention is not invariant, but rather is dependent on the level of the to-be-attended information.
\end{abstract}

Keywords Selective attention - Working memory ·

Cognitive and attentional control

It is well established that the availability of cognitive resources affects the efficiency of visual selective attention. Previous research has demonstrated that temporarily limiting the availability of cognitive resources by imposing concurrent external load (e.g., Lavie, Hirst, de Fockert, \& Viding, 2004) impedes the success with which the distracting influence of irrelevant information is overcome. As a result, interference effects from irrelevant distractors are greater when working memory (WM) load is high (as compared to low).

L. Ahmed $(\bowtie) \cdot$ J. W. de Fockert

Department of Psychology Goldsmiths, University of London,

New Cross,

London SE14 6NW, UK

e-mail: lubnalubna@hotmail.com
Typically, this detrimental influence of cognitive load on the ability to prevent distraction has been shown in tasks requiring selective attention to local information and the avoidance of peripheral irrelevant information. For example, Lavie et al. (2004) found that the distracting influence of a peripheral irrelevant letter, when attending to a centrally presented target letter, is greater when WM load is high than when it is low (see also de Fockert \& Wu, 2009). However, visual information often comprises local-level information that is subsumed within a larger holistic pattern, such as a face or scene. Whilst the impact of WM load on locally attended selection is well established, the impact of WM load on the capacity to selectively attend to information at a more global level, whilst ignoring local-level information, is not known. Here we used hierarchical Navon-type stimuli (Navon, 1977) to investigate the impact of WM load on selective attention to different levels of the same stimulus.

Hierarchical stimuli consist of a large global shape made up of smaller local shapes. When attention is directed to one of the levels, the degree to which relevant information is successfully attended to is quantified by the performance difference (congruency effect) between trials when the information at the unattended level is the same as (congruent trials) or different from (incongruent trials) the attended information. Measuring congruency effects as a function of WM load during attention to either local- or globallevel information thus enables investigating the effect of WM on selective attention at these two levels. The effects of WM load on distractor processing so far have been shown in tasks in which the relevant and irrelevant forms of information are presented as distinct stimuli, rather than at different levels of the same stimulus, as in Navon-type stimuli. Our first objective was to investigate whether the effect of WM load increasing distractibility during attention to a local target would be replicated when observers attend to the local level and ignore the global level of a hierarchical stimulus 
(Exp. 1). We then investigated whether increasing WM load has the same effect when attention is directed to the global level and information at the local level has to be ignored (Exp. 2). We also replicated these effects by measuring both local and global interference in the same participants (Exp. 3).

Load theory (Lavie et al., 2004) suggests that cognitive resources are required to maintain distinctions between processed relevant and irrelevant information. The efficiency of this process is compromised when resources are depleted, as under high WM load, resulting in greater distraction from irrelevant information. Thus, according to load theory, the congruency effects should be greater during high (vs. low) WM load in all conditions, provided that the effect of WM load on distractor processing when attending to local information in flanker-like paradigms is replicated when relevant and irrelevant information is compounded within the same hierarchical stimulus. The well-established global precedence for information processing (see Navon, 1977; Poirel, Pineau, \& Mellet, 2008) may result in an asymmetrical influence of WM load on distraction in the local and global attention conditions: The more distracting global-level information may be harder to ignore under high WM load than the less distracting local information. Nevertheless, load theory predicts an increase in distraction effects as a function of WM load, regardless of whether the distractors appear at a local or a global level of processing.

Recent findings, however, offer an alternative prediction regarding the effect of WM load on interference effects from local distractors when attending to the global level. When interference effects were plotted as a function of the spatial separation between the target and the distractor in a flanker task, the results suggested that the distribution of spatial attention becomes more dispersed when WM load is high (Caparos \& Linnell, 2010). High WM load seems to reduce the ability to effectively constrain attention to relevant local information, leading to more distraction from peripheral distractors. In the present Navon task, such an effect of WM load would lead to a shift towards global processing. This would mean that when attending to the local level, the irrelevant global level would produce more interference under high WM load. In contrast, when attending to the global level, the irrelevant local level would produce less interference with increasing load.

\section{Experiment 1}

Method

Participants A group of 18 university students (two males; age: $M=19.60, S D=5.86$ ) were tested individually, in exchange for course credit or payment. All had normal or corrected-to-normal vision.
Apparatus The participants were tested using E-Prime software (Schneider, Eschman, \& Zuccolotto, 2002) in a dimly lit testing cubicle, seated at a viewing distance of approximately $50 \mathrm{~cm}$ from a $21 \times 28 \mathrm{~cm}$ computer monitor. Responses were collected with a standard computer keyboard.

Stimuli All stimuli were presented in white on a black background. Each trial consisted of a WM task and a letter task. For the WM task, a six-digit memory set, subtending a visual angle of $9.5^{\circ}$ horizontally, was presented centrally in 18-point Courier New font. A single-digit memory probe was presented at the end of each trial. For the letter task, the displays were Navon-type letters " $\mathrm{H}$ " and "S," which consisted of a large (global) letter made up of small (local) letters (see Fig. 1). The local and global letters were the same on congruent displays and different on incongruent displays. The large $\mathrm{H}$ contained 19 small letters, and the large S contained 25 small letters. Each large-letter type contained seven small letters across the vertical and horizontal axes, thus ensuring that the spacing between the local elements and the size ratio between the local and global elements were equal for both global letters. The large letters subtended approximately $6.27^{\circ}$ vertically and $5.71^{\circ}$ horizontally. The small letters subtended approximately $0.34^{\circ} \times$ $0.34^{\circ}$. To encourage trial-by-trial refocusing, and to avoid static spatial attention to a predictable stimulus location, the letter displays were randomly presented in one of four possible locations, either top left or right or bottom left or right, with respect to fixation. ${ }^{1}$ The distance from the central fixation point to the centre of the hierarchical stimulus was $6.84^{\circ}$, and the distance was $3.20^{\circ}$ to the nearest point of the large letter. These visual angles were consistent for all positions and stimulus types.

Procedure See Fig. 1 for example trial sequences. First, the WM digit set was presented for $3,000 \mathrm{~ms}$, followed by a 250 ms blank screen. In the high-WM-load condition, the six digits were presented in a random nonsequential order. In the lowWM-load condition, the six digits were always in ascending sequential order. At the end of the trial, a single memory probe digit with a question mark was presented for $5,300 \mathrm{~ms}$ or until response. The memory probe was equally likely to have been present or absent in the set, and when present it was equally likely to have been presented at any of the six memory set positions. Half the participants were instructed to press the " $\mathrm{w}$ " key if they thought that the probe had been present in the

\footnotetext{
${ }^{1}$ Two $2 \times 2 \times 4 \times 2$ ANOVAs were conducted on the mean correct-RT and accuracy data, with the factors Congruency (congruent, incongruent), WM Load (low, or high), Stimulus Location $(1,2,3,4)$ as withinsubjects factors and Attention Level (local, global) as a betweensubjects factor. Neither the main effect of stimulus location nor any interactions were significant ( $p>1.6$ in all cases). We therefore collapsed the data across stimulus locations for the main analysis.
} 
Fig. 1 Illustration of the trial sequence for each condition of working memory load. The enlarged hierarchical letters are examples of congruent and incongruent global S stimuli used in Experiments 1 and 2 and in Experiment 3, respectively. The images are not to scale
High WM Load trial

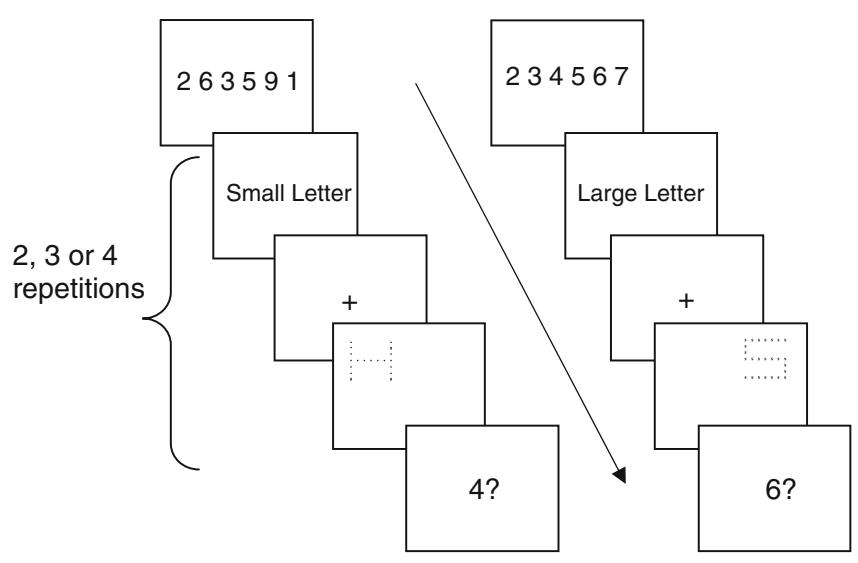

Experiment 1 \& 2

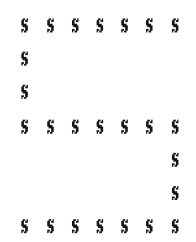

Experiment 3

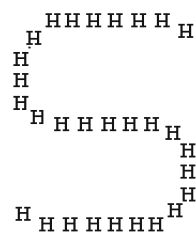

memory set for the trial, and the "s" key if they thought it had not, using the middle and index fingers of the left hand, respectively. Response mappings were the opposite for the other half. Key allocations were counterbalanced across participants. Feedback following incorrect or absent responses to the memory task was given with a 200 -ms red cross.

The letter task was presented between the WM set and memory probe displays. A series of two, three, or four letter trials were presented during each WM trial, to ensure that the presentation of the memory probe was unpredictable, thus encouraging active rehearsal of the WM set during the entire trial (see de Fockert, Rees, Frith, \& Lavie, 2001). The message "Next trial" was displayed for 2,500 ms between WM trials.

For the letter task, a written cue ("large letter" or "small letter") subtending $7^{\circ}$ horizontally was presented for $1,500 \mathrm{~ms}$ to indicate the to-be-attended level for the trial. The cued level did not change within a WM trial. The ratio of local- to globaldirected trials was $4: 1{ }^{2}$ A central fixation cross was then presented for $1,500 \mathrm{~ms}$, followed by the letter stimulus for $200 \mathrm{~ms}$, and finally a blank response interval lasting up to $1,800 \mathrm{~ms}$. The task was to indicate whether the letter on the cued level was either an $\mathrm{H}$ or an $\mathrm{S}$ as quickly and accurately as possible, by using the index and middle fingers of the right hand to press the "0" key on the numeric keypad if the target letter was an $\mathrm{H}$, and the "2" key if it was an S. Key allocations were counterbalanced across participants. The feedback following incorrect or absent responses was the same as in the WM task.

A block comprised $34 \mathrm{WM}$ trials and 88 letter trials, presented in randomised order. Each of the four stimulus types (congruent and incongruent $\mathrm{H}$ and $\mathrm{S}$ ) appeared equally often in each of the four possible locations within a block.

\footnotetext{
${ }^{2}$ Previous work has shown that congruency effects from the irrelevant level are reduced when attention is consistently directed to a particular level rather than switched between levels (e.g., Lamb \& Yund, 1996; Ward, 1982). Therefore, a small number of trials on which attention had to be directed to the level other than the one used on the majority of trials (global in Exp. 1, local in Exp. 2) were included in each block.
}

Four experimental blocks were presented in total. WM load was manipulated between blocks, separated by breaks, and participants were informed of the WM load type at the start of each block. Blocks of low and high WM load were presented in alternating order, which was counterbalanced across participants. Across each experiment, participants performed 72 congruent and 72 incongruent local-directed trials and 16 congruent and 16 incongruent global-directed trials in each WM load condition. Participants first completed two practice blocks, each consisting of four low- and high-WM-load trials and between six and 12 letter trials.

\section{Results}

WM task A mean error rate was computed for each participant as a function of WM load (low, high) and was analysed in a paired-samples $t$ test. $^{3}$ Probe error rates were lower under low $(M=12 \%)$ than under high $(M=17 \%) \mathrm{WM}$ load, $t(17)=2.19, p<.043$.

Letter task Data were only analysed for the local-directed trials. Letter responses were excluded from the analysis if there was an error in response to the memory probe for that trial. In addition, for the letter reaction time (RT) analysis, letter responses that were inaccurate or faster than $200 \mathrm{~ms}$ were also excluded. The mean correct RT and error rate were computed for each participant as a function of congruency and WM load (Table 1) and were analysed in two-way ANOVAs.

The RT ANOVA revealed a main effect of congruency, $F(1,17)=6.50, p<.021, \eta^{2}=.277$ : Participants were

\footnotetext{
${ }^{3}$ Paired-sample $t$ test analysis of the mean RTs revealed that they did not vary as a function of WM load ( $p>.3$ in all experiments). Accuracy, rather than speed, was emphasised to participants for the memory probe response; thus, the higher error rates under high than under low WM load in all experiments confirmed that the load manipulation was successful.
} 
Table 1 Mean error rates and correct reaction times (in milliseconds) on the letter identification task as a function of congruency type and working memory (WM) load across the three experiments

\begin{tabular}{|c|c|c|c|c|c|c|}
\hline & \multicolumn{3}{|c|}{ Low WM Load } & \multicolumn{3}{|c|}{ High WM Load } \\
\hline & Congruent & Incongruent & Cong. Effect & Congruent & Incongruent & Cong. Effect \\
\hline \multicolumn{7}{|l|}{ Error Rates } \\
\hline Exp. 1 (Local attend) & $.06(.01)$ & $.06(.01)$ & $.00(.04)$ & $.06(.01)$ & $.10(.01)$ & $.04(.01)$ \\
\hline Exp. 2 (Global attend) & $.05(.01)$ & $.10(.01)$ & $.06(.01)$ & $.07(.01)$ & $.16(.01)$ & $.08(.01)$ \\
\hline Exp. 3 (Local attend) & $.13(.02)$ & $.19(.03)$ & $.07(.02)$ & $.11(.02)$ & $.19(.03)$ & $.08(.02)$ \\
\hline Exp. 3 (Global attend) & $.09(.01)$ & $.14(.02)$ & $.06(.02)$ & $.10(.01)$ & $.17(.02)$ & $.07(.02)$ \\
\hline \multicolumn{7}{|l|}{ Reaction Times } \\
\hline Exp. 1 (Local attend) & $892(43)$ & $903(36)$ & $11(12)$ & $878(40)$ & 919 (37) & $41(10)$ \\
\hline Exp. 2 (Global attend) & $800(36)$ & $855(41)$ & $55(16)$ & $867(44)$ & $859(38)$ & $-8(13)$ \\
\hline Exp. 3 (Local attend) & $444(22)$ & $492(23)$ & $48(9)$ & 448 (19) & $512(19)$ & $64(7)$ \\
\hline Exp. 3 (Global attend) & $355(24)$ & $393(30)$ & $38(11)$ & $376(25)$ & $400(30)$ & $23(12)$ \\
\hline
\end{tabular}

Values in parentheses are standard errors

slower when responding to incongruent $(M=911 \mathrm{~ms})$ than to congruent $(M=885 \mathrm{~ms})$ trials. The main effect of WM load was not reliable $(p>9)$. Critically, the interaction between congruency and $\mathrm{WM}$ load was reliable, $F(1,17)=10.23$, $p<.005, \eta^{2}=.376$, and as is illustrated in Fig. 2, was due to a larger congruency effect under high $(M=41 \mathrm{~ms})$ than under the low $(M=11 \mathrm{~ms}) \mathrm{WM}$ load.

The error rate ANOVA revealed that participants made more errors when responding to incongruent $(M=18 \%)$ than to congruent $(M=16 \%)$ trials, $F(1,17)=4.77, p<.043, \eta^{2}=$ .219 , and made more errors under high $(M=18 \%)$ than under low $(M=16 \%)$ WM load, $F(1,17)=6.87, p<.018, \eta^{2}=.288$. The Congruency $\times$ WM Load interaction was again significant, $F(1,17)=5.92, p<.026, \eta^{2}=.259$, and was due to a

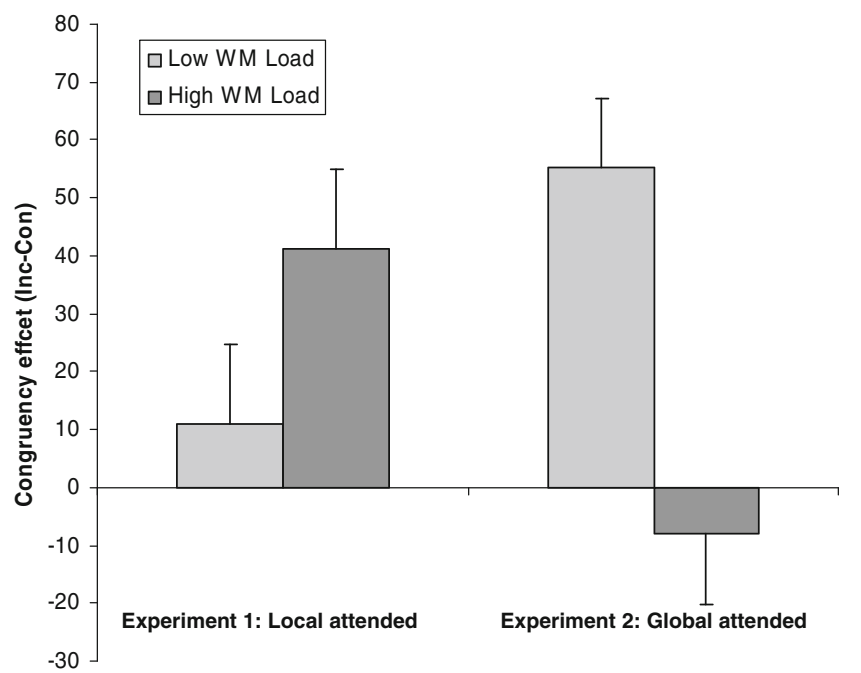

Fig. 2 Mean reaction time (RT) congruency effects (in milliseconds) as a function of level of attention and working memory (WM) load in Experiments 1 and 2. Error bars represent between-subjects standard errors larger congruency effect in the high $(M=0.04 \%)$ than in the low $(M=0 \%)$ WM load condition.

\section{Experiment 2}

In Experiment 2, we asked participants to attend to the global level to record the effect of WM load when observers selectively attended to the global, rather than the local, information. Thus, whereas Experiment 1 investigated the effect of WM load on interference from a global distractor during attention to a local target, in Experiment 2 we measured the effect of WM load on interference from a local distractor during attention to a global target.

\section{Method}

Participants A group of 18 university students (six males; age: $M=21.93, S D=7.83$ ) were tested individually, in exchange for course credit or payment. All had normal or corrected-to-normal vision. None had participated in Experiment 1.

Stimuli and procedure In Experiment 2, the ratio of trials with a local or a global cue was reversed, so that participants attended to the global level of the hierarchical stimuli on the majority of trials. All other aspects were as in Experiment 1.

\section{Results}

$W M$ task Probe error rates were again lower under low $(M=$ $9 \%)$ than under high $(M=15 \%)$ WM load, $t(17)=2.41, p<$ .028 (see note 3 ). 
Letter task Data were only analysed for global-directed trials. The same trial exclusion and data computation criteria were applied as in Experiment 1. The mean correct-RT and error rate data (Table 1) were analysed in two-way ANOVAs.

The RT ANOVA revealed a main effect of congruency, $F(1,17)=4.79, p<.043, \eta^{2}=.220$; participants were slower when responding to incongruent $(M=857 \mathrm{~ms})$ than to congruent $(M=833)$ trials. There was also a main effect of WM load, $F(1,17)=5.59, p<.03, \eta^{2}=.248$; responses were slower under high $(M=863 \mathrm{~ms})$ than under low $(M=827 \mathrm{~ms})$ WM load. The interaction between congruency and WM load was reliable, $F(1,17)=10.59, p<.005, \eta^{2}=.384$. As is illustrated in Fig. 2, and in contrast to Experiment 1, the interaction was due to a smaller congruency effect under high $(M=-8 \mathrm{~ms})$ than under low $(M=55 \mathrm{~ms}) \mathrm{WM}$ load.

The error rate ANOVA revealed that participants made more errors when responding to incongruent $(M=13 \%)$ than to congruent $(M=6 \%)$ trials, $F(1,17)=31.15, p<.001$, $\eta^{2}=.661$, and made more errors on high $(M=11 \%)$ than on low $(M=8 \%)$ WM load trials, $F(1,17)=12.64, p<.002$, $\eta^{2}=.426$. The Congruency $\times$ WM Load interaction was not significant in the error rates.

To confirm that the congruency effects were indeed different depending on whether attention was directed to the local (Exp. 1) or the global (Exp. 2) level, a three-way mixed ANOVA was conducted with Congruency (congruent, incongruent) and WM Load (low, high) as within-subjects factors and Attention Level (local, global) as a between-subjects factor. In the RTs, there was a strong three-way interaction, $F(1,34)=18.69, p<.001, \eta^{2}=.355$. This confirmed that WM load had a different effect on congruency effects, depending on the level of the hierarchical stimulus that was attended. In the error rates, the three-way interaction was not reliable $(p>.2)$.

\section{Discussion: Experiments 1 and 2}

In Experiment 1, when attending to the local level of the hierarchical stimulus, the interfering effect of the irrelevant global level increased as a function of WM load; both accuracy and RT congruency effects were larger during high- than during low-WM-load conditions. This effect replicates similar findings from selective attention tasks in which the target and distractor items were different stimuli (e.g., Lavie \& de Fockert, 2005; Lavie et al., 2004) and also when the relevant and irrelevant information constituted distinct stimuli that were nonetheless spatially overlapping (e.g., de Fockert et al., 2001). These results demonstrate for the first time that increasing WM load increases distractor effects from the irrelevant global level when attending to local elements in hierarchical stimuli.
By contrast, the findings in Experiment 2 show that WM load has the opposite effect, of reducing distraction from the irrelevant local level, as the congruency effects were smaller under high than under low WM load when attending to the global information. The finding suggests that participants were more efficient when selectively attending to relevant information during high WM load in this case.

Although the opposing effects of WM load on distraction from the local and global levels were statistically reliable, this result has to be considered with caution, as it was obtained in two different participant groups. Since individuals are known to vary considerably in terms of selective attention efficiency (e.g., Kane \& Engle, 2003), we ran Experiment 3 in an attempt to replicate the findings from Experiments 1 and 2, this time manipulating local versus global attention within the same participants.

\section{Experiment 3}

In Experiment 3, we again measured the effects of WM load on distraction from local and global processing levels, this time using a within-subjects design. We made two further modifications in order to address the issue that, in contrast with previous findings (Navon, 1977; Poirel et al., 2008), there was no evidence for a global precedence effect in Experiments 1 and 2. The global precedence effect is characterised by a global advantage (RTs are faster when responding to this level) and a greater global interference effect (global irrelevant information interferes more than local irrelevant information; Kimchi, 1992; Navon, 2003). In Experiment 3, we therefore added a condition without additional WM load in order obtain a baseline measure of local and global interference on our task. In addition, to promote global precedence, we increased the salience of the global form by increasing the number of local elements contained within each global shape (Kinchla \& Wolfe, 1979).

\section{Method}

Participants A group of 26 university students (seven males; age: $M=18.69, S D=0.83$ ) were tested individually in exchange for course credit. All had normal or correctedto-normal vision, and none had participated in Experiment 1 or 2 .

Stimuli The stimuli were slightly modified to promote global precedence. The numbers of small letters contained in the large $\mathrm{H}$ and S letters were increased from 19 to 24 and from 25 to 29 , respectively, and the corners of the large S were 
rounded to form a more conventional S shape (Fig. 1). All other stimulus details were as in Experiment 1.

Procedure The procedure was similar to that of Experiment 1. No-load blocks were identical to the WM load blocks, but without the WM components. The experiment was conducted across two testing sessions scheduled two weeks apart. The participants completed two no-load blocks at the start of each session, followed by either the local- or the global-directed blocks of low and high WM load. The order of presentation of global/local attention was counterbalanced across participants. To reduce testing times, the fixation cross and ISI presentations were reduced from the values of 1,500 and 2,500 ms in Experiments 1 and 2 to 500 and $1,000 \mathrm{~ms}$, respectively, in Experiment 3.

\section{Results}

Data were only analysed for trials with the level to be attended to in each condition. The same exclusion criteria were applied as in Experiment 1, and mean correct RT and error rates were computed as in Experiment 1 (Table 1).

\section{No-load blocks}

We first established that interference effects showed the expected global precedence by performing a two-way ANOVA on mean correct RTs in the no-load condition, with Attention Level and Congruency as within-subjects factors. We found main effects of congruency $[F(1,25)=26.27, p<$ $\left..001, \eta^{2}=.512\right]$ and attention level $[F(1,25)=25.54, p<$ $\left..001, \eta^{2}=.505\right]$. RTs were slower when responding to incongruent $(M=421 \mathrm{~ms})$ than to congruent $(M=379 \mathrm{~ms})$ trials, and were faster on global $(M=364)$ than on local ( $M=453 \mathrm{~ms}$ ) directed trials, reflecting the global advantage effect. In line with the anticipated greater global interference effect, the congruency effects were larger on local $(52 \mathrm{~ms})$ than on global $(32 \mathrm{~ms})$ trials; however, this characteristic of the global precedence effect failed to reach significance $\left(F=2.17, p=.15, \eta^{2}=.08\right)$.

The error rate ANOVA revealed a main effect of congruency $\left[F(1,25)=17.12, p<.001, \eta^{2}=.406\right]$ : More errors were made on incongruent $(M=20 \%)$ than on congruent $(M=12 \%)$ trials. The remaining effects were not reliable.

\section{WM load blocks}

WM task Probe error rates were significantly lower under low $(M=13 \%)$ than under high $(M=19 \%)$ WM load, $t(25)=$ $4.36, p<.001$ (see note 3 ).
Letter task A three-way fully within-subjects ANOVA was conducted on mean correct RTs, with the factors Congruency (congruent, incongruent), Attention Level (local, global), and WM Load (no, low and high). There was a main effect of congruency $\left[F(1,25)=47.56, p<.001, \eta^{2}=.655\right]$; RTs were slower on incongruent $(M=440 \mathrm{~ms})$ than on congruent $(M=$ $397 \mathrm{~ms}$ ) trials. The main effect of WM load was not reliable $(p=.14)$. The main effect of attention level $[F(1,25)=$ $\left.34.78, p<.001, \eta^{2}=.582\right]$ and the two-way interaction between attention level and congruency $[F(1,25)=5.07$, $\left.p=.033, \eta^{2}=.169\right]$ were both significant. RTs were faster on global- $(M=369)$ than on local- $(M=467 \mathrm{~ms})$ directed trials, and the congruency effect was larger at the local $(55 \mathrm{~ms})$ than at the global $(31 \mathrm{~ms})$ attention level. Thus, both characteristics of the global precedence effect were evident here.

The two-way interactions between attention level and WM load $(p=.75)$ and congruency and WM load $(p=.96)$ were not reliable. Critically, the three-way interaction was significant $\left[F(1,25)=3.44, p=.049, \eta^{2}=.223\right]$, indicating that the effects of WM load on distraction from the irrelevant levels differed when attending to the local or the global level.

Two follow-up ANOVAs were conducted to assess the effects driving the three-way interaction. First, we conducted a 2 (congruency) $\times 2$ (attention level) $\times 2($ WM load: no vs. low WM load) ANOVA. The analysis revealed main effects of congruency $\left[F(1,25)=39.40, p<.001, \eta^{2}=.612\right]$ and attention level $\left[F(1,25)=34.78, p<.001, \eta^{2}=.582\right]$. No other main effects or interactions were significant. The three-way interaction was also not reliable $(p>.5)$, indicating that when WM load was low or absent, distraction from the irrelevant levels was the same when attending to either local or global levels.

Next, we conducted a similar 2 (congruency) $\times 2$ (attention level) $\times 2$ (WM load: low vs. high WM load) ANOVA to compare the low- and high-WM-load conditions. Once again, there were main effects of congruency $[F(1,25)=$ 59.94, $\left.p<.001, \eta^{2}=.706\right]$ and attention level $[F(1,25)=$ $\left.28.21, p<.001, \eta^{2}=.530\right]$, but the main effect of WM load was not significant $(p=.14)$. We did find a two-way interaction between attention level and congruency $[F(1,25)=$ 4.36, $\left.p=.047, \eta^{2}=.149\right]$ : The congruency effect was greater at the local $(56 \mathrm{~ms})$ than at the global $(31 \mathrm{~ms})$ attention level. The two-way interaction between attention level and WM load was not significant $(p=.84)$.

The key finding was a significant three-way interaction $\left[F(1,25)=5.97, p<.02, \eta^{2}=.193\right]$, which followed the same pattern as across Experiments 1 and 2. The congruency effect increased with WM load when attending to the local level (from 47 to $64 \mathrm{~ms}$ ), and in contrast, decreased as a function of WM load during the global-attend trials (from 38 to $23 \mathrm{~ms}$; see Fig. 3). Although $t$ tests of the difference in 


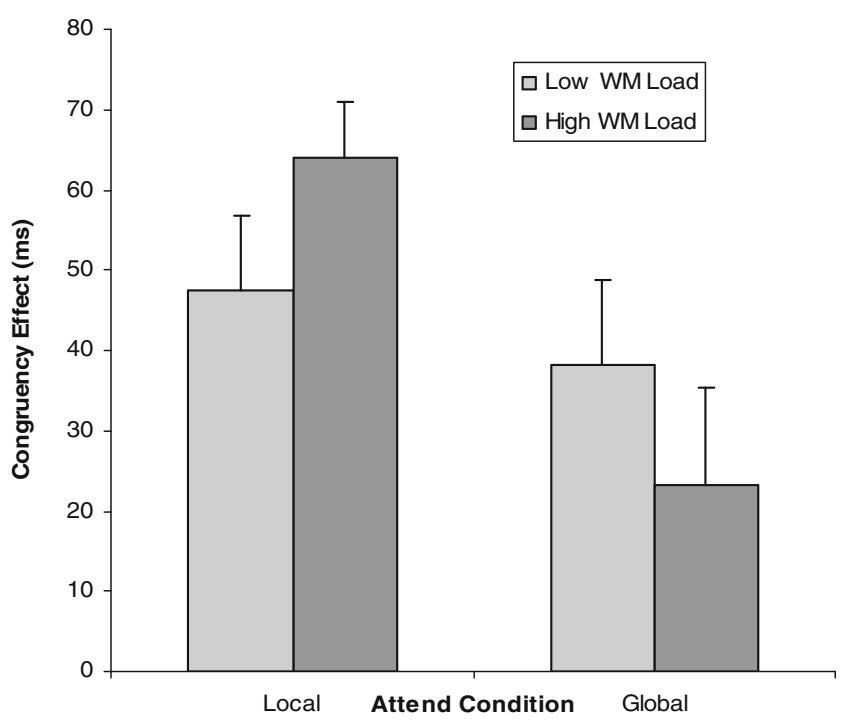

Fig. 3 Mean reaction time (RT) congruency effects (in milliseconds) as a function of level of attention and working memory (WM) load in Experiment 3. Error bars represent within-subjects standard errors

the congruency effects as a function of WM were just short of significance under both local attention $[t(25)=1.52, p=.07$, one-tailed $]$ and global attention $[t(25)=1.35, p=.09$, onetailed], the significant crossover interaction confirmed that the directions of the congruency effects as a function WM load were opposite under local versus global attention.

The $2 \times 2 \times 3$ ANOVA on the error rates revealed corresponding effects on the RTs analysis for the main effects of congruency $\left[F(1,25)=18.00, p<.001, \eta^{2}=.419\right]$ and attention level $\left[F(1,25)=4.58, p=.042, \eta^{2}=.155\right]$. The twoway interaction between attention level and WM load was also significant $\left[F(1,25)=3.86, p=.035, \eta^{2}=.243\right]$ and was due to a slight decrease in error rates as a function of WM load during local attention (WM load: no, $M=19 \%$; low, $M=17 \%$; high, $M=16 \%$ ), but not during global attention (WM load: no, $M=$ $16 \%$, low, $M=12 \%$; high, $M=14 \%$ ). No other main effects and interactions, including the three-way interaction, were reliable in the error rates.

\section{Discussion: Experiment 3}

In Experiment 3, we again found that the effects of loading WM on distractibility were opposite during attention to the local versus the global level of a hierarchical stimulus. Whereas interference from the global level during attention to the local level was increased by greater WM load (as in Lavie et al., 2004), interference from the local level during attention to the global level was reduced as WM load increased.

Crucially, these opposing effects were replicated in a paradigm soliciting the classic global precedence effect. In Experiment 3, the overall latencies were faster to global than to local targets in the no-load condition, and global distractors produced greater interference than did local distractors. The pattern of interference effects did not vary significantly between the no-load and low-WM-load conditions. This suggests that our low-WM-load condition can be regarded as a valid baseline for assessing interference effects without concurrent WM load.

Furthermore, these opposing effects of WM load on local/global distraction were obtained within the same participants in Experiment 3, suggesting that the pattern of effects across Experiments 1 and 2 was not due to individual differences in attentional selectivity between the groups. Finally, the effects of WM load on interference were found in the absence of any effects of WM load on absolute RTs.

\section{General discussion}

Our findings show that the effect of increasing WM load on selective attention efficiency when processing hierarchical stimuli is not invariant, but rather depends critically on the level of the stimulus that has to be attended. Increasing WM load impeded the ability to effectively attend to relevant information on the local level. In contrast, when attending to the global level, selective attention was better when concurrent WM load was high as compared to low.

These results present two original findings. Firstly, they demonstrate for the first time that increasing WM load can increase distractor effects in hierarchical stimuli in certain conditions. Similar to previous findings from selectiveattention tasks in which the target and distractor items constituted different stimuli (e.g., Lavie et al., 2004, Lavie $\&$ de Fockert, 2005) and also when relevant and irrelevant information constituted distinct stimuli that were spatially overlapping (e.g., de Fockert et al., 2001), we found that WM load increased interference effects from the irrelevant global level when attending to local-level information in Experiments 1 and 3. The second, and more important, novel finding was that such an increase in distractibility as a function of WM load may be restricted to situations of local processing (e.g., Lavie et al., 2004). High WM load did not increase distractibility from the local level when attention had to be directed to the global level in Experiments 2 and 3. Instead, an unprecedented decrease in distractibility with cognitive load occurred in this case, when the distractors occurred at the local level.

The present findings are compatible with growing evidence that suggests that the spatial distribution of attention affects distractibility. For example, attentional capture from peripheral singletons is greater when the task requirements solicit diffuse rather than focused attention (Belopolsky \& Theeuwes, 2010; Belopolsky, Zwaan, Theeuwes, \& Kramer, 2007; for data slightly incompatible with this explanation, 
see also Santangelo, Botta, Lupiáñez, \& Spence, 2011). Moreover attentional capture from irrelevant colour singletons is greater when the concurrent WM load is high (vs. low; Lavie \& de Fockert, 2005). Such a finding may be explained in terms of a greater spatial dispersion of attention under high WM load, leading to a greater tendency for singletons to capture attention.

Evidence for this view from a difference perspective has been presented by Caparos and Linnell (2010), who measured the interference caused by distractors at incrementally greater distances from the target to map the distribution of the attentional window under low and high WM load in a flanker-like paradigm. The findings suggested that the distribution of the attentional window is more dispersed when WM load is high versus low. Measuring distractor effects from the local and global levels in the Navon paradigm as a function of WM load provides an alternative test for the proposed shift towards global processing with load. In the Navon task, when attending to the local level, interference effects from the irrelevant global level are expected to increase with WM load, as a dispersion of spatial attention with WM load would cause attention to be shifted towards the distracting global level, in this case. When attending to the global level and ignoring the local level, on the other hand, additional WM load is expected to have the same effect of making it less likely that attention will be constrained to the local level; however, in this case this would have the opposite effect, of reducing interference from the local-level distractors. This is exactly what we found, and thus the present findings provide further evidence that high WM load leads to a dispersion in spatial attention.

The spatial dispersion of attention is inferred from our findings in terms of the relative interference effects from local versus global levels of processing. As we did not explicitly measure attentional dispersion, alternative explanations could account for these findings. One possibility is that the present WM task induced local processing under high load (when attention had to be strictly focused on the order of the memory set items) and more global processing under low WM load (when attention could directed more globally towards the entire set). Consequently, local (vs. global) processing would be relatively compromised during the subsequent selective-attention task under high (vs. low) WM load, leading to the pattern of interference effects that we obtained. Further work will be required to distinguish between these explanations.

Our findings add to the growing body of literature indicating that the effect of WM load on selective attention is not always to increase distractibility, but that reduced distractibility can also result from increasing WM load, in certain circumstances (e.g., Dittrich \& Stahl, 2012; Kim, Kim, \& Chun, 2005; Park, Kim, \& Chun, 2007). For example, whether high WM load increases or reduces distractor effects can depend on whether the WM task overlaps more with target processing (in which case, greater interference is the result) or distractor processing (Kim et al., 2005; Park et al., 2007). Our findings show that another factor that determines whether increasing WM load will increase or reduce distractibility is whether attention is directed to local-level information (leading to more interference from the global level) or to global-level information (leading to less interference from the local level).

The present findings highlight that by investigating WM load effects in tasks that require local-directed attention to a target (e.g., de Fockert et al., 2001, Lavie \& de Fockert, 2005; Lavie et al., 2004), previous research on the role of WM in selective attention has so far only shown part of the picture. In visual processing, attention often needs to switch between local and global levels of processing, depending on the current task demands. For example, when using a satellite navigation device while driving a car, the information on the navigation screen needs to be processed at a detailed local level, whereas attention to the traffic environment requires a more global level of analysis. Using stimuli for which the relevant target information could occur at either the local or the global level, we were able to show that WM load has opposing effects on distractibility depending on the attended level of processing, thus uncovering a more comprehensive account of the impact of WM load on the distribution of attention. These findings suggest that the role of WM in selective attention may not just be to maintain clear priorities between relevant and irrelevant processing (Lavie et al., 2004), but also to actively constrain attention to the local level.

\section{References}

Belopolsky, A. V., \& Theeuwes, J. (2010). No capture outside the attentional window. Vision Research, 50, 2543-2550. doi:10.1016/ j.visres.2010.08.023

Belopolsky, A. V., Zwaan, L., Theeuwes, J., \& Kramer, A. F. (2007). The size of an attentional window modulates attentional capture by color singletons. Psychonomic Bulletin \& Review, 14, 934 938. doi:10.3758/BF03194124

Caparos, S., \& Linnell, K. J. (2010). The spatial focus of attention is controlled at perceptual and cognitive levels. Journal of Experimental Psychology. Human Perception and Performance, 36, 1080-1107.

de Fockert, J. W., Rees, G., Frith, C. D., \& Lavie, N. (2001). The role of working memory in visual selective attention. Science, 291, 1803-1806. doi:10.1126/science. 1056496

de Fockert, J., \& Wu, S. (2009). High working memory load leads to more Ebbinghaus illusion. European Journal of Cognitive Psychology, 21, 961-970.

Dittrich, K., \& Stahl, C. (2012). Selective impairment of auditory selective attention under concurrent cognitive load. Journal of Experimental Psychology. Human Perception and Performance, $38,618-627$.

Kane, M. J., \& Engle, R. W. (2003). Working-memory capacity and the control of attention: The contributions of goal neglect, response 
competition, and task set to Stroop interference. Journal of Experimental Psychology. General, 132, 47-70. doi:10.1037/00963445.132.1.47

Kim, S.-Y., Kim, M.-S., \& Chun, M. M. (2005). Concurrent working memory load can reduce distraction. Proceedings of the National Academy of Sciences, 102, 16524-16529. doi:10.1073/ pnas.0505454102

Kimchi, R. (1992). Primacy of wholistic processing and global/local paradigm: A critical review. Psychological Bulletin, 112, 24-38. doi:10.1037/0033-2909.112.1.24

Kinchla, R. A., \& Wolfe, J. M. (1979). The order of visual processing: "Top-down", "bottom-up", or "middle-out". Perception \& Psychophysics, 25, 225-231. doi:10.3758/BF03202991

Lamb, M. R., \& Yund, E. W. (1996). Spatial frequency and attention: Effects of level-, target, and location-repetition on the processing of global and local forms. Perception \& Psychophysics, 58, 363373.

Lavie, N., \& de Fockert, J. W. (2005). The role of working memory in attentional capture. Psychonomic Bulletin \& Review, 12, 669-674.

Lavie, N., Hirst, A., de Fockert, J. W., \& Viding, E. (2004). Load theory of selective attention and cognitive control. Journal of
Experimental Psychology. General, 133, 339-354. doi:10.1037/ 0096-3445.133.3.339

Navon, D. (1977). Forest before trees: The precedence of global features in visual perception. Cognitive Psychology, 9, 353-383.

Navon, D. (2003). What does a compound letter tell the psychologist's mind? Acta Psychologica, 114, 273-309.

Park, S.-J., Kim, M.-S., \& Chun, M. M. (2007). Concurrent working memory load can facilitate selective attention: Evidence for specialized load. Journal of Experimental Psychology. Human Perception and Performance, 33, 1062-1075.

Poirel, N., Pineau, A., \& Mellet, E. (2008). What does the nature of the stimuli tell us about the global precedence effect? Acta Psychologica, 127, 1-11.

Santangelo, V., Botta, F., Lupiáñez, J., \& Spence, C. (2011). The timecourse of attentional capture under dual-task conditions. Attention, Perception, \& Psychophysics, 73, 15-23.

Schneider, W., Eschman, A., \& Zuccolotto, A. (2002). E-Prime user's guide. Pittsburgh: Psychology Software Tools.

Ward, L. M. (1982). Determinants of attention to local and global features of visual forms. Journal of Experimental Psychology. Human Perception and Performance, 8, 562-581. 